\title{
Evaluation of a pilot service to facilitate discharge of patients with stable long-term mental health needs from secondary to primary care: the role of Primary Care Mental Health Specialists
}

\author{
Kate Hamilton-West ${ }^{1}$, Sarah Hotham ${ }^{1}$, Wei Yang ${ }^{2}$, Julie Hedayioglu ${ }^{1}$ and Charlotte Brigden ${ }^{1}$ \\ ${ }^{1}$ Centre for Health Services Studies (CHSS), University of Kent, Canterbury, UK \\ ${ }^{2}$ Department of Global Health and Social Medicine, Strand Campus, King's College London, Strand, London WC2R 2LS, UK
}

\begin{abstract}
Aim: We aimed to evaluate a pilot service to facilitate discharge of patients with stable long-term mental health needs from secondary to primary care. Background: Patients with stable long-term mental health conditions are often not discharged from secondary mental health services when no longer needed due to insufficient systems and processes to enable safe, effective, recovery-focussed treatment and support. The Primary Care Mental Health Specialist (PCMHS) Service was developed to address this gap; new PCMHS posts were introduced to act as a conduit for patients being discharged from secondary care and a single point of referral back into secondary care, should it be required. The two-year pilot, across six Clinical Commissioning Groups in South East England, began in March 2013. Methods: Interviews were conducted with all PCMHS employed in the pilot service $(n=13)$ and a sample of service users $(n=12)$. The views of professionals working alongside the service, including GPs, Psychiatrists and Mental Health Nurses, were captured using a brief online questionnaire $(n=50)$. Time and Activity Recording Sheets were used to capture data required for economic analysis. Findings: Our findings indicate that the service is working well from the perspective of patients; staff employed within the service and professionals working alongside the service. Patients described the service as a 'safety net' they could fall back on in case of difficulties, whereas staff used the analogy of a 'bridge' to describe the way the service improved communication and collaboration between the various professionals and organisations involved in the patient's care. Improvements in well-being were seen to result from increased support for those transitioning from secondary to primary care, a more pro-active approach to relapse prevention and increased engagement in daily activities. Each PCMHS covered 36 patients in a one-month period, with a unit cost of $£ 73.01$ per patient.
\end{abstract}

Key words: mental health; primary care; psychiatric care; service evaluation

Received 13 October 2016; revised 13 March 2017; accepted 17 March 2017; first published online 18 April 2017

The global burden of mental health disorders is considerable and growing, with significant impacts on health and well-being, as well major social and economic consequences (World Health

Correspondence to: Dr Kate Hamilton-West, Centre for Health Services Studies, George Allen Wing, University of Kent, Canterbury CT2 7NF, UK. Email: k.e.hamilton-west@kent.ac.uk
Organisation, 2016). In the United Kingdom, Government strategy No Health without Mental Health emphasises the need for improved prevention, detection and treatment of mental health disorders, as well as greater choice, control and personalisation of mental health care (Department of Health, 2011). At the same time services are required to achieve significant cost efficiency savings

(C) Cambridge University Press 2017 
through initiatives such as the UK National Health Service (NHS) Quality, Innovation, Productivity and Prevention programme, implementation of Any Qualified Provider and Payment by Results $(P b R)$ as set out in Equity and Excellence: Liberating the NHS (Department of Health, 2010).

The Health and Social Care Act (2012) gave rise to a number of important structural changes, including the establishment of Clinical Commissioning Groups (CCGs), which are held to account for the outcomes they achieve, including mental health outcomes, through the Commissioning Outcomes Framework (2011, NHS Commissioning Board). The Act places statutory duties on CCGs to promote continuous improvement in the quality of health services, with particular regard to clinical effectiveness, patient experience and patient safety. In 2012-2013 mental health services were brought within the scope of $\mathrm{PbR}$, requiring that patients are assessed by their mental health provider and allocated to a cluster, which forms the basis of the contracting arrangements between commissioners and providers. These clusters must then be reviewed regularly in line with the timing and protocols set out in the mental health clustering booklet (Department of Health, 2013).

Development of the Primary Care Mental Health Specialist (PCMHS) Service reflected the recommendations of the Joint Commissioning Panel for Mental Health (2012), which emphasised the need to deliver services that enable safe, effective recovery-focussed treatment and support for people with stable long-term mental health conditions. It was recognised that patients were often not discharged from secondary mental health care when no longer needed, because systems and processes were not in place to provide adequate support and that this had an impact upstream on responsiveness to urgent referrals challenges which have also been highlighted by the recently established Independent Commission on Acute Adult Psychiatric Care (Crisp et al., 2016). New PCMHS posts were introduced to act as a conduit for patients being discharged from secondary care and a single point of referral back into secondary care, should it be required. Introduction of the new role was intended to improve mental health capacity and expertise in primary care and increase provision of primary care mental health services, based on local population need. The two-year pilot began in
March 2013 and covered six CCGs in South East England. The service evaluation reported here aimed to capture experiences of patients accessing the pilot service, as well as views of frontline staff delivering the service and other health professions working alongside the service. We also aimed to assess the economic cost of the new service.

\section{Methods}

\section{Patient and public involvement}

A local service user led 'Experts-by-Experience' group advised on the evaluation methods, including the development of materials (consent form, information sheet and interview topic guides) and practical considerations (eg, where patient interviews could take place). Methods were also developed with input from the $\mathrm{CCG}$ and Commissioning Support Unit (CSU), who advised on the information required for evaluation of the pilot service.

\section{Participants and procedure}

For the purpose of the pilot, people with stable long-term mental health conditions were defined as those allocated to clusters 7, 11 and 12 of the national $\mathrm{PbR}$ framework, currently in receipt of secondary care mental health services and with a likely diagnosis (International Classification of Diseases-10) of schizophrenia, bipolar disorder, recurrent depression and chronic neurotic, stressrelated and somatoform disorders.

Patients were recruited from three of the six CCGs included in the evaluation (including the earliest and most recent areas to implement the pilot). They were provided with an information sheet and invitation letter by their PCMHS. Those interested in participating sent their contact details to the evaluation team in a stamped addressed envelope. We then made contact to arrange a convenient time for the interview to be conducted, either by telephone, or face-to-face. We asked that all service users in the three CCGs $(n=172)$ receive an invitation letter; 20 patients expressed an interest in being interviewed, three dropped out before the interview due to deteriorating mental health and five did not respond to messages left by the evaluation team. A final sample of 12 service users (five male, seven female, age range 
23-64 years, $M=50.8$ years) was interviewed and responses analysed.

PCMHS were seconded ${ }^{1}$ from their NHS Trust for the duration of the pilot and were community psychiatric nurses (CPN) or occupational therapists (OT) by profession. They were hosted by GP practices or local mental health provider organisations. All host organisations granted permission for the evaluation team to approach PCMHS to discuss participation. PCMHS were provided with information sheets before consenting to be interviewed. All PCMHS employed in the pilot service across the six CCGs agreed to take part and subsequently participated in face-to-face interviews $(n=13)$. For the economic analysis, a subsample of 11 PCMHS was selected to record time spent on specific activities.

Information about the evaluation was distributed to staff with experience working alongside the pilot service via PCMHS, CCGs and host organisations, along with a link to an online questionnaire. In total, 50 respondents completed the questionnaire, including GPs $(n=16)$, Managers/Operations $(n=11)$, Primary Care Link Workers $(n=7)$, Psychiatrists $(n=6)$, Mental Health Nurses $(n=3)$, Practice Managers $(n=2)$ and Social Workers $(n=1)$; three respondents indicated their profession as 'other'.

Interviews were conducted between September 2013 and May 2014. Questionnaire responses were gathered between June and November 2014. Economic data were recorded between 1 and 31 October 2014.

\section{Design}

This is a mixed-method formative evaluation (Dehar et al., 1993) that captured impact in both outcomes and processes. A formative evaluation was considered most appropriate as the evaluation was intended to cover the early implementation of the pilot and provide information to inform decision making regarding ongoing service provision. Unit cost analysis (Drummond et al., 2005) was considered most appropriate for the economic evaluation, as this provides information on the

\footnotetext{
${ }^{1}$ The term 'seconded' means that the employees are assigned to work, for a temporary period of time, for another host organisation. On expiry of a 'secondment', employees are able to return to their original employer.
}

amount of additional resource needed for a patient to receive an intervention; this can help commissioners and policy makers decide whether it is possible to implement the intervention on a large scale given the resources available.

\section{Materials}

\section{Topic guides}

Topic guides were developed by the evaluation team with input from the Experts-by-Experience group, CCG and CSU. Topic guides for the patient interviews explored experiences of the service and impacts on their mental health and well-being. Topic guides for the PCMHS interviews focussed on experiences of implementing the service.

\section{Online questionnaire}

A brief online questionnaire was developed specifically for this evaluation, using the secure Qualtrics $^{\circledR}$ system. Respondents were asked to select their profession and CCG area, to indicate whether they were aware of the pilot service (Yes/ No) and had experience working with a PCMHS (Yes/No). These items were included to check that the data captured represented the views of professionals with experience of the pilot service. Respondents were then asked to indicate whether, in their experience, the PCMHS role worked well (scale ranging from $1=$ strongly disagree, to $5=$ strongly agree), if they considered that patients benefited from the service (Yes/No) and if improvements could be made to the service (Yes/No). They were invited to provide further comments (including specific examples) in relation to each question using a free text response format.

\section{Time and Activity Recording Sheets (TARS)}

TARS were provided to enable PCMHS to record data required for the economic analysis, including time spent on main activity (ie, contact with patients) and additional activities (eg, liaison activities and events organised by the CCGs), travel costs associated with these activities and monthly income.

\section{Ethical considerations}

An evaluation proposal was submitted to the Research Management and Governance Consortium 
before recruitment and we were advised that ethical review by an NHS Research Ethics Committee would not be necessary, as the project met Health Research Authority criteria for evaluation/audit, rather than research. ${ }^{2}$ However, ethical principles were adhered to regarding data confidentiality, informed consent and right to withdraw. Participants had the opportunity to ask questions before providing signed consent and participating in interviews. They were reminded that they were not required to answer any questions they felt uncomfortable with and were free to terminate the interview at any point, without giving a reason. Patients were further informed that their decision would not affect any services they were receiving. Participants were assured that only the evaluation team would have access to their data, interview data would be not be stored together with names, addresses or telephone numbers and individuals would not be identified in any reports. Electronic data were stored on a password protected database at the Centre for Health Services Studies (CHSS), University of Kent; hardcopies were stored in a locked filing cabinet at CHSS.

\section{Data analysis}

Interviews were recorded, transcribed verbatim and entered into the NVivo software package. Data were analysed using the Framework Method (Ritchie and Spencer, 2011). One member of the team read through all interview transcripts and coded responses according to themes identified from the interview guide, before developing subthemes by identifying common responses. The transcripts were then read by a second member of the team, who reviewed the themes and subthemes; differences in coding were identified and consensus reached by discussion.

Questionnaire data were entered into SPSS (Version 22). Descriptive statistics were calculated for quantitative variables. A content analysis technique was used to categorise responses to the open-ended questions. Responses were broadly organised into themes by one member of the team and reviewed by a second member. Again, differences in categorisation were discussed and a consensus reached.

\footnotetext{
${ }^{2}$ http://www.hra.nhs.uk/research-community/before-you-apply/ determine-whether-your-study-is-research/
}

Time and activity data were analysed using STATA 13 software. Unit cost analysis was performed from a health service perspective. Hence, only costs associated with implementing the service were considered; the analysis did not take into account costs relating to societal inputs (eg, carer support).

\section{Results}

\section{Patient interviews}

Three initial themes were identified from the interview guide: (1) participants' experiences of the service; (2) impacts of the service on patient mental health and well-being; and (3) overall reflections on the service.

\section{Patients' experiences of the service}

Three subthemes were identified in relation to patients' experiences of the service; how the service differed from previous experience of mental health care; benefits of the service and disappointments with the service.

In relation to the first subtheme, participants described greater continuity of care under the new service, which allowed for a trusting relationship to develop between the patient and PCMHS and prevented a constant repeating of information to clinical staff. Patients also reported that the care they received from the PCMHS was more tailored to their needs than previous care. They reported feeling understood by the PCMHS and more involved in decisions about their care than they had in the past:

They (PCMHS) can basically tell when they come in the door I'm having a bad time or I'm up. You do need to have somebody who can read that rather than maybe somebody you've seen once in a few months and then they seem to think they know everything about you and they don't.

Patients reported that the main benefit of the service was that it contributed to the prevention of relapses; having consistent support in place, where this had been lacking in the past, served as a 'safety net'. Accessibility was also a key benefit cited; patients reported that they could rely on 
PCMHS to return calls promptly and they could be contacted at any time. The support provided was viewed by patients as useful, relevant and important to the improvement of their mental health; PCMHS were seen as empathetic and understanding of mental health issues - experts in their field:

I think it's the dignity with which you're treated. Nobody patronises me or assumes that I just can't cope or anything like that, it's just sensitive help at the right time.

Overall, patients spoke with high regard about the service and its impacts on their health and wellbeing. However, they reported disappointment with two aspects: the length of time it took to receive appointment letters and difficulty being referred into the service:

My disappointment was actually finding out it existed and my battle with the GP to get it but once I've been having that, no not at all. I would have liked to have known it existed to even enquire whether I'd be eligible for it; yeah it was very tucked away at the time.

(P002)

\section{Impacts of the service on patient mental health and} well-being

Patients reported a wide range of impacts on their mental health and well-being including reduced levels of anxiety/stress, feeling less lonely, more confident about coping with their mental health condition and more optimistic about the future. They reported greater confidence in their ability to undertake practical tasks, as well as increased engagement in daily activities and improved health. Patients also described feeling that a burden had been lifted since accessing the service:

I think increasingly I'm looking forward to the future rather than when I was in my lowest time, I always treated the tomorrow as the last day. And I didn't always know how to be happy or to be sad about it because I was in such a mess, but now I see myself as having a future.

(P017)
And also getting out and about a lot more and doing different things, things I hadn't tried before with the help of the PCMHS.

(P011)

.... but because I'm dealing better with the mental health side of my condition, it does help me to deal with the physical side of the conditions.

\section{Overall reflections on the service}

All patients interviewed indicated they were very satisfied with the service. They did not make specific suggestions for improvement, but did express concerns about removal of the service and how this could impact their mental health:

I think it's been excellent. They, I've always been treated with a great deal of sort of courtesy and professionalism. I've never felt embarrassed or that I was a nuisance, quite the reverse and it's all worked extremely well to not just keep me out of hospital but to keep me you know well in the community.

Well I would hope that they would keep going because I think I would fall apart if it was just left to me on my own. As long as they don't just start leaving me stranded somewhere thinking 'Oh she can cope now and get on with it'.

\section{PCMHS interviews}

Three initial themes were identified from the topic guide: (1) main responsibilities and working models; (2) evaluation of service implementation and patient outcomes; (3) challenges of implementing the service.

\section{Main responsibilities and working models}

Three subthemes were identified from PCMHS descriptions of their key areas of work: building relationships with other health professionals and organisations; identifying patients suitable for discharge to the new service; and conducting therapy work with patients. 
In relation to the first subtheme, PCMHS discussed the importance of building relationships with GPs, practice managers, care co-ordinators, community mental health and secondary care teams. They described how they offered advice to GPs about mental health diagnoses and supported them with patient advice and signposting. Relationships were also built outside the NHS for example, with third sector organisations and community support groups. PCMHS also described their role in identifying patients who could benefit from the service:

What we will do with GPs is as goodwill
because we're really trying to build up you
know links with the GP, occasionally ring us
up and say look we've got a client here, we're
really not too sure what to do, could you just
come in and give us some advice, so we are
starting to do some assessments.

Patients were identified via discussion with both GPs and secondary care teams. PCMHS highlighted some initial challenges accessing patients in secondary care, although this became easier as relationships with secondary care teams developed:

We're attending the meetings of the teams (based in secondary care) and we're pushing, saying look, look at your $7,11 \& 12 \ldots$ highlight who you think may benefit from sort of coming out with us and we'll come in, we'll do the bulk of the work, let's get a CPA arranged and we'll get them out.

PCMHS reported undertaking a range of therapy work, including psychoeducation (eg, on medication, diagnosis and relapse), help with practical tasks (eg, completing forms) and providing patients with tools to organise and structure their lives (eg, through support offered by OT). They reported that therapy work was person-centred and recovery-focused; the aim was to reduce social isolation, develop a sense of purposefulness and prevent readmission to secondary care:

To try and help them move forward with their recovery, so spend a bit more time on the things that they mightn't have had time to develop in secondary services like looking at maybe voluntary work, further education and just to try and help them a bit further along in their recovery.

\section{Evaluation of service implementation and patient outcomes}

All PCMHS highlighted positive impacts on patient outcomes. Four subthemes were identified: prevention of relapse; speed of response; providing a bridge; and providing appropriate care.

Relapse prevention was achieved by developing trusting relationships with patients, educating them to recognise signs of relapse and encouraging patients to make contact before a crisis develops:

You educate them anyway about their relapse signs. That's the first thing you do. You let the patients know what the signs (are) you ask them and also their carers.

(P013)

This was supported by the ability of the service to react quickly to changing situations, which had not been possible in the past:

It would probably take them a week to get an appointment with the GP, and then for the GP to write the letter, and then for the letter to get to [the Trust] and if they weren't high risk then another probably week to two weeks before they would get an appointment. That's almost a month which somebody with mental health, a month can be a long, long time.

(P006)

PCMHS worked to bridge gaps between different organisations and between care providers and patients, helping to facilitate communication, improve collaborative working and reduce patients' concerns about discharge from secondary care:

I say it's about being that bridge, you've got someone the GPs can talk to, you've got somebody the voluntary services can talk to, you've got somebody that [the Trust] can talk to, so it kind of helps. Instead of it being very in their own sort of bubbles you sort of help them communicate.

Key elements of appropriate care provision included: developing meaningful relationships with 
patients; proactively addressing mental health needs before these escalated; and seeing patients in the home environment or local GP surgery, which was considered less stigmatising than secondary mental health services:

We're normalising their mental health, we're seeing them somewhere where they're comfortable.

\section{Challenges of implementing the service}

PCMHS highlighted three key challenges in implementing the service. First, in the early stages of the pilot they experienced difficulties accessing patients' primary and secondary care records, although this improved as awareness of the service increased. Second, PCMHS highlighted challenges accommodating patients with additional requirements, such as those with social care packages and/or requiring depot injections, due to lack of appropriate arrangements and/or equipment. Finally, concerns were expressed regarding workload and capacity to expand in the future, with PCMHS highlighting the challenge of balancing administrative duties with a growing clinical caseload:

I think a lot of the issues that most people have are not to do with the work itself, not the kind of clinical contact, it's more the kind of pressures that you get you know through typically your paperwork or stuff that you kind of have to constantly have to update, which feels less integral to your role and you can spend literally $70 \%$ of your time updating Rio and $30 \%$ of your time seeing clients.

(P002)

\section{Analysis of responses from other professional groups}

The vast majority of participants ( $n=46,92 \%)$ agreed or strongly agreed that the PCMHS role works well, whereas $48(96 \%)$ agreed that patients benefited from the support provided by the PCMHS; 40 respondents $(80 \%)$ agreed that improvements could be made to the service. Free text responses are summarised below.

\section{Do you think the PCMHS role works well?}

Most participants $(n=44,88 \%)$ provided free text comments. Only one commented on dissatisfaction with the service, highlighting a lack of contact with the PCMHS as the reason. Reasons for satisfaction with the service related to five main themes: preventing relapse and/or readmission to secondary care; patient satisfaction with the service; increased opportunities to work across professional groups; providing support in community settings and bridging the gap between primary and secondary mental health care. Example quotes are provided below:

It simply prevents relapse through early detection of relapse indicators. The primary care specialist kept closely monitoring and we worked together to prevent relapse and rehospitalisation.

(P024, Psychiatrist)

By attending one of our team meetings and giving a clear explanation of their role and how they fit in with secondary and primary care. Our counsellors felt supported by them, and, as a team, felt we had a named person to consult with should issues arise.

(P043, Operations Manager)

I have patients who do not want to go to secondary care mental health service because they did not have good experience with the service. However they were very receptive to receive support from the primary care mental health specialist in the community.

(P045, GP)

Have patients benefited from the support provided by the PCMHS?

Free text responses were provided by 42 respondents. Two indicated that they selected 'no' because they did not have sufficient knowledge of the service to comment. The remaining comments described ways in which patients benefited from the service. Three main benefits were described: providing care in community settings; bridging the gap between primary and secondary mental health care and improving patient empowerment and quality of life:

... Individuals are taking responsibility of their own recovery and linking in with community resources.

(P003, Operations/Management) 
A service user was discharged to shared care. This has worked well for the service user and feels more empowered.

(P015, Member of Community Mental Health Team)

Many (patients) have avoided eviction and entered the community in a positive way.

(P032, Inclusion worker)

\section{Could the service be improved?}

Free text responses were provided by 39 participants. Comments indicated that the service could be improved by: increasing the number of PCMHS, widening PCMHS job descriptions/ responsibilities (eg, to see patients not allocated to clusters 7, 11 and 12) and improving practical elements of the pilot (eg, information technology systems and protocols):

With more practitioners this service may be able to take referrals from primary care reducing referrals to secondary services.

(P025, Mental Health Nurse)

To expand the role and not just to restrict them for clusters 7,11 \& 12.I hope they will be able to see patients with complex needs that they are not in those specific clusters, (but are) too complicated for GPs \& IAPT services to look after and do not meet the criteria for secondary mental health services.

(P009, GP)

It would work better if they had joint access to Rio to ensure seamless handovers and better communications.

$(\mathrm{P} 030, \mathrm{CPN})$

\section{Economic analysis}

A total of 399 patients received the service during October 2014. Each specialist covered 36 patients and worked an average of 16.64 days (137.14 hours). The average cost for 1 hour of PCMHS time was $£ 15.95$.

Table 1 shows the average time taken to screen a new patient, deliver a patient session and attend additional events, together with unit costs per month.

Figure 1 shows the typical cost breakdown per session. Contact hours accounted for the largest proportion of costs associated with delivering a patient session, followed by administrative tasks, then travel to and from sessions.

The monthly unit cost per patient for delivering patient consultation sessions is $£ 73.01$; this is based on the average monthly unit cost for each CCG. The monthly unit cost per PCMHS for delivering additional events is $£ 607.41$.

\section{Conclusions}

Data collected for this evaluation indicate that the service is working well from the perspective of patients; staff employed within the service and professionals working alongside the service. All three groups described improvements in patient care and benefits for mental health and well-being. Specifically, participants described patient care as more person-centred and more easily accessible than in the past; they also highlighted greater continuity of care resulting from the introduction of the PCMHS role. Improvements in patient mental health and well-being were seen to result from increased support for those transitioning from secondary mental health services to primary care as well as a more proactive approach to relapse prevention and support

Table 1 Average time taken to screen a new patient, deliver a patient session and attend additional events, together with unit costs per month (based on data from 1 to 31 October 2014)

\begin{tabular}{lllr}
\hline & Number & Time (hours) & Unit cost (f) \\
\hline Screening new patients & 7 patients & 1.4 hours per new patient & f20.92 \\
Delivering sessions & 36.36 sessions per PCMHS per month & 2.93 hours per session & f41.28 \\
Additional events & 3.45 sessions per PCMHS per month & 6.98 hours per session & f121.87 \\
\hline
\end{tabular}

Costs for delivering sessions include contact time, travel, time for preparation and administration. Additional events include training, meetings with GPs and Clinical Commissioning Group and other relevant events that specialists are required to attend as part of their job, but not relating to a particular patient.

PCMHS = Primary Care Mental Health Specialist. 


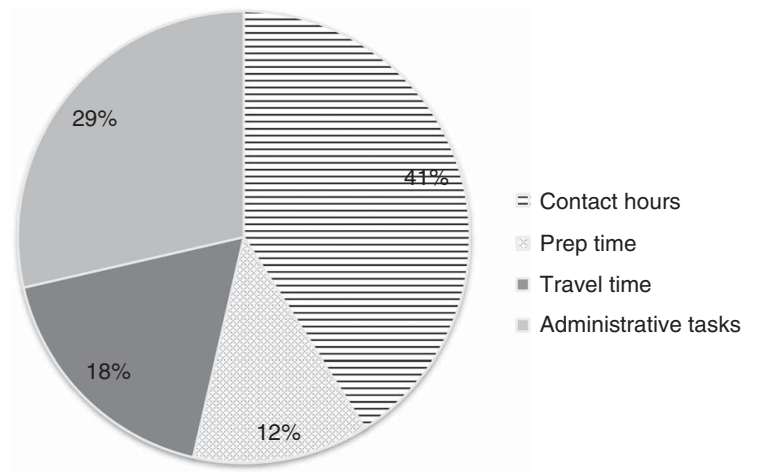

Figure 1 Cost breakdown (\%) per consultation session with patient

for engaging in daily activities. Patients described the service as a 'safety net' they could fall back on in case of difficulties, whereas staff used the analogy of a 'bridge' to describe the way the service improved communication and collaboration between the various professionals and organisations involved in the patient's care, as well as between services and patients. The service also enabled patients to better understand and cope with their mental health condition, with benefits for empowerment and quality of life.

It is notable that the accounts of all three groups were so similar. We did not encounter reports of major failings or concerns about the service; rather, comments regarding areas for improvement tended to relate to a desire for the service/role to be expanded. These comments are particularly noteworthy given the wider context in which this service is delivered - for example, the Five Year Forward View for Mental Health (Mental Health Taskforce, 2016) highlights a number of challenges facing NHS mental health services, including lack of access to physical health care in people with mental health problems, many of whom do not know who is responsible for co-ordinating their care and have not agreed what care they should receive with a clinician. The report also highlights that people with severe and prolonged mental illness are at risk of dying on average 15-20 years earlier than those without mental health problems - one of the greatest health inequalities in England. As data for the PCMHS evaluation were drawn from six CCGs, it is evident that benefits are not limited to a particular area, service or team. Hence, findings suggest that the approach has the potential to be rolled out on a wider scale across the NHS.

The economic analysis presented here could be used to assist commissioners and providers in planning and delivery of similar services - PCMHS working at a cost of $£ 15.95$ per hour were able to provide care for 36 patients within a one-month period. Although additional events organised by the CCGs increased the monthly cost of delivering the service, these are likely to be important for delivery of safe, effective care - future research could examine this in greater detail, considering the nature, duration and frequency of training and related activities necessary to deliver a service of this nature.

A number of limitations should be noted. First, although we were able to capture the views of all staff employed in the service, interviews were conducted with only 12 patients, of a potential sample of 172. Invitation letters were distributed by PCMHS and it was not possible to monitor whether all service users received an invitation (as the evaluation team did not have access to patient records). Hence, findings may represent the views of a subsample of patients, with positive experience of the service. We recommend that future research/evaluation work in this area adopts a different recruitment strategy, with invitation letters distributed by staff not involved in the delivery of the service. Second, although participants reported benefits in terms of relapse prevention, we were not able to examine this directly. Future research could compare frequency of relapse/ readmission to secondary mental health services before and after introduction of a PCMHS service, or compare data between areas with and without PCMHS posts. Third, economic data relates to a one-month period during 2014 - hence, costs would need to be updated for the purpose of planning the implementation and delivery of similar services. Finally, the pilot was conducted over a two-year period, starting in 2013 - hence, new services based on the PCMHS pilot will need to be adapted in line with recent, and ongoing, changes to the provision of mental health/primary care services.

Notwithstanding these limitations, the current evaluation provides support for the introduction of PCMHS roles as a means of enabling safe, effective, recovery-focussed treatment and support for people with stable long-term mental health 
conditions and facilitating discharge from secondary mental health care. We were unable to identify published evaluations of other similar services and recommend further research to examine the impact of this type of support on patient outcomes, as well as work to examine the potential for this approach to be rolled out more widely.

\section{Acknowledgements}

The authors would like to thank Dr Jan Smith and Nichola Chisnall for assistance with data collection. The authors would also like to thank the CCGs, CSU, staff and service users for their invaluable contributions to this project.

\section{Financial Support}

This evaluation was funded by MCCH $(£ 30,000)$.

\section{Conflicts of Interest}

None.

\section{References}

Commissioning Outcomes Framework 2011: NHS Commissioning Board. Retrieved 28 September 2016 from https://www. england.nhs.uk/wp-content/uploads/2011/11/COF-final1.pdf.

Crisp, N., Smith, G. and Nicholson, K. (editors) 2016. Old problems, new solutions - improving acute psychiatric care for adults in England. London: The Commission on Acute Adult Psychiatric Care. Retrieved 28 September 2016 from http://www.rcpsych. ac.uk/pdf/Old_Problems_New_Solutions_CAAPC_Report_ England.pdf.
Dehar, M., Casswell, S. and Duignan, P. 1993: Formative and process evaluation of health promotion and disease prevention programs. Evaluation Review 17, 204-20.

Department of Health 2010: Equity and excellence: liberating the NHS. Retrieved 28 September 2016 from https://www.gov.uk/ government/uploads/system/uploads/attachment_data/file/ 213823/dh_117794.pdf.

Department of Health 2011: No health without mental health: a cross government health outcomes strategy for people of all ages. Retrieved 28 September 2016 from https://www.gov. uk/government/uploads/system/uploads/attachment_data/file/ 213761/dh_124058.pdf.

Department of Health 2013: Mental health payment by results guidance for 2013-14. Retrieved 28 September 2016 from https:/www.gov.uk/government/uploads/system/uploads/ attachment_data/file/232162/Mental_Health_PbR_Guidance_ for_2013-14.pdf.

Drummond, M., Sculpher, M., Torrance, G., O'Brien, B. and Stoddart, G. 2005. Methods for the economic evaluation of health care programmes. Oxford: Oxford University Press.

Health and Social Care Act 2012: London: Stationery Office. Retrieved 28 September 2016 from http://www.legislation. gov.uk/ukpga/2012/7/pdfs/ukpga_20120007_en.pdf.

Joint Commissioning Panel for Mental Health 2012: Guidance for commissioners of primary mental health care services. Volume 2: practical mental health commissioning. Retrieved 28 September 2016 from http://www.rcpsych.ac.uk/.

Mental Health Taskforce 2016: Five year forward view for mental health. Retrieved 16 January 2017 from https://www.england. nhs.uk/wp-content/uploads/2016/02/Mental-Health-TaskforceFYFV-final.pdf.

Ritchie, J. and Spencer, L. 2011: Qualitative data analysis for applied policy research. 173-194. In Bryman, A. and Burgess, R.G., editors Analyzing qualitative data. London: Routledge, 173-94.

World Health Organisation 2016: Mental disorders factsheet. Retrieved 28 September 2016 from http://www.who.int/ mediacentre/factsheets/fs396/en/. 